\title{
Tunable magnetic properties in ultrathin Co/garnet heterostructures
}

M. Pashkevich, A. Stupakiewicz' , A. Kirilyuk, A. Maziewski, A. Stognij, N. Novitskii, A. Kimel, and Th. Rasing

Citation: Journal of Applied Physics 111, 023913 (2012); doi: 10.1063/1.3679548

View online: http://dx.doi.org/10.1063/1.3679548

View Table of Contents: http://aip.scitation.org/toc/jap/111/2

Published by the American Institute of Physics 


\title{
Tunable magnetic properties in ultrathin Co/garnet heterostructures
}

\author{
M. Pashkevich, ${ }^{1,2}$ A. Stupakiewicz, ${ }^{1, a)}$ A. Kirilyuk, ${ }^{3}$ A. Maziewski, ${ }^{1}$ A. Stognij, ${ }^{2}$ N. Novitskii, ${ }^{2}$ \\ A. Kimel, ${ }^{3}$ and Th. Rasing ${ }^{3}$ \\ ${ }^{1}$ Laboratory of Magnetism, Faculty of Physics, University of Bialystok, Lipowa 41, Bialystok, Poland \\ ${ }^{2}$ Scientific-Practical Materials Research Centre of the NASB, P. Brovki 19, Minsk, Belarus \\ ${ }^{3}$ Radboud University Nijmegen, Institute for Molecules and Materials, Heyendaalseweg 135, \\ Nijmegen, Netherlands
}

(Received 22 September 2011; revised 27 December 2011; published online 30 January 2012)

\begin{abstract}
We demonstrate how the magnetic properties of metal/dielectric Co/yttrium iron garnet heterostructures can be engineered by both changing the garnet thickness and adding an ultrathin Co cover layer. The observed magnetization reversal process in the heterostructures is explained by both cubic and perpendicular growth-induced magnetic anisotropy of the garnet films. In particular, the perpendicular magnetic anisotropy can be strongly increased for reduced thickness. A strong influence of a $2 \mathrm{~nm}$ Co layer on the domain structure geometry and magnetization processes has been found for $1.8 \mu \mathrm{m}$ garnet films. (C) 2012 American Institute of Physics. [doi:10.1063/1.3679548]
\end{abstract}

\section{INTRODUCTION}

In the last decade, numerous studies of magnetic multilayers and heterostructures were motivated by their importance for applications in devices. This area of nanomagnetism has strongly developed, due to new growth techniques and lithographic processes. For fundamental research, such heterostructures give the unique possibility to engineer high quality two-dimensional interfaces and create phenomena which do not exist in a bulk material. On the other hand, new functionalities may emerge from the coexistence of two materials with complementary properties, such as magnetism and ferroelectricity, metallic and dielectric, antiferromagnetic and ferromagnetic, etc. An interesting combination is formed by a metallic ferromagnetic thin film on top of a dielectric ferrimagnetic garnet based on yttrium iron garnet (YIG). The functionality of YIG systems has been shown to be very broad, with examples such as the excitation of surface plasmons, ${ }^{1}$ the propagation of nonlinear spin-waves, ${ }^{2}$ Bose-Einstein condensation of a magnon gas, ${ }^{3}$ high-temperature photomagnetism, ${ }^{4}$ the observation of the inverse Faraday effect induced by an ultrafast laser pulse, ${ }^{5}$ and many others. A combination of a metal layer on a garnet system may create the possibility to modify different properties. Recently, it was reported that ion beam-sputtered Fe films on a 100-nm-thick YIG layer possess a perpendicular magnetic anisotropy. ${ }^{6}$ In the thickness range between 5 and $10 \mathrm{~nm}$, the stripe domain structure of YIG was transferred into the Fe films, due to the presence of strong interlayer exchange coupling. ${ }^{6}$ Static and dynamic properties were also investigated for a $30 \mathrm{~nm}$ permalloy film on a $0.5 \mu \mathrm{m}$ $(\mathrm{YBiLu})_{3}(\mathrm{FeAl})_{5} \mathrm{O}_{12}$ layer that is characterized by a perpendicular anisotropy. ${ }^{7}$ A strong direct exchange coupling is revealed via the formation of enlarged closure domains with a preferred orientation at the interface between the permalloy film and the garnet layer. As a result, the domain pattern of such a heterostructure shows an increased zero-field stripe

\footnotetext{
${ }^{\text {a) }}$ Author to whom correspondence should be addressed. Electronic mail: and@uwb.edu.pl.
}

period in comparison to the parent garnet layer. ${ }^{7}$ The magnetization reversal process and magnetic domain structure were the focus point of these studies. YIG films with iron partially substituted with $\mathrm{Co}^{2+}$ and $\mathrm{Co}^{3+}$ ions ${ }^{8}$ show interesting magnetic properties, such as several spin-reorientation phase transitions in a temperature range of $20-300 \mathrm{~K}^{9}$ and both quasistatic $^{10}$ and ultrafast ${ }^{11}$ light-induced changes in magnetic anisotropy. Light pulses excite large-angle magnetization precession in such garnets, the phase and the amplitude of the precession being determined by the polarization of the light. If coupled with a nanostructure ferromagnetic (metallic) overlayer, such photomagnetic effects in the garnet may also be transferred to the overlayer, thus creating new possibilities for ultrafast switching.

In the present paper, we report on magnetization reversal, domain structures, and magnetic anisotropy in ultrathin Co films grown by ion beam sputtering on Co-substituted garnet films with different thicknesses. We observe a strong change of uniaxial magnetic anisotropy after decreasing the initial garnet thickness as well as a modification of the domain structure in a garnet covered by a magnetostatically coupled $2 \mathrm{~nm}$ Co layer.

\section{SAMPLE PREPARATION}

Experimental studies were performed on Co/garnet heterostructures and reference garnet films. The initial 5.8- $\mu \mathrm{m}-$ thick garnet film of $\mathrm{Y}_{2} \mathrm{CaFe}_{3.9} \mathrm{Co}_{0.1} \mathrm{GeO}_{12}$ composition was grown using liquid phase epitaxy on a paramagnetic substrate $\mathrm{Gd}_{3} \mathrm{Ga}_{5} \mathrm{O}_{12}(\mathrm{GGG})$ in plane (001). ${ }^{8}$ The miscut angle of the GGG substrate has been about $0.1^{\circ}$. The preparation conditions and some crystallographic properties of the films were published elsewhere. ${ }^{12}$ The crystal chemistry of magnetic garnets has been extensively studied in the past. ${ }^{13}$ In our sample, the vibrating sample magnetometer study gave, at room temperature saturation magnetization, $4 \pi \mathrm{M}_{\mathrm{S}}=88 \mathrm{G}$ and the Neel temperature $T_{N}=445 \mathrm{~K}$. Afterwards, the sample was thinned down to $1.8 \mu \mathrm{m}$ by argon ion beam sputtering in the low energy regime with an etching rate of about 


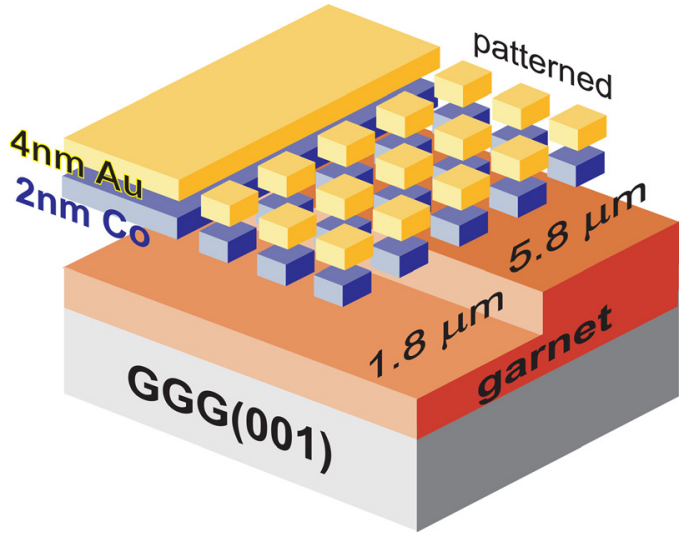

FIG. 1. (Color online) Schematic configuration of the sample, including the $20 \times 20 \mu \mathrm{m}$ pattern area on the garnet parts.

$0.83 \mu \mathrm{m} / \mathrm{h}$. Prior to the metallic layer deposition, the surface of the garnet film was smoothed by a low energy oxygen ion beam. $\mathrm{Au}(4 \mathrm{~nm}) / \mathrm{Co}(2 \mathrm{~nm})$ bilayers were grown in situ on both 1.8 and $5.8 \mu \mathrm{m}$ YIG films by ion-beam sputter deposition. ${ }^{14}$ A polycrystalline Co layer was grown on top of an unperturbed garnet film. Figure 1 illustrates the sample configuration. The gold layer was used to prevent the Co layer from oxidation. A $20 \times 20 \mu \mathrm{m} \mathrm{Au} /$ Co pattern, for comparison of coupling between $\mathrm{Co}$ and garnet films and domain structures' modifications on garnets, was fabricated by liftoff photo-lithography. The photoresist was patterned on the garnet films prior to the Co layer deposition. The dose of UV exposure and the development process were precisely controlled to result in a sharp edge profile of resist patterns. After deposition of the $\mathrm{Au} / \mathrm{Co}$ heterostructure, a lift-off procedure was performed to remove the previously unexposed resist.

\section{RESULTS AND DISCUSSION}

The process of magnetization reversal was studied via magneto-optical Faraday hysteresis measurements using a perpendicular magnetic field $\mathrm{H}_{\perp}$. Measurements were performed by laser light with a wavelength of $690 \mathrm{~nm}$ and a spot diameter of approximately $0.1 \mathrm{~mm}$. A modulation technique with a photoelastic modulator was used for determination of the Faraday rotation angle. The hysteresis loops for different garnet thicknesses and Co/garnet films, after separation of the paramagnetic contribution to the Faraday rotation from the GGG substrate, are shown in Fig. 2. The shape of the hysteresis loops observed for the initial $5.8-\mu \mathrm{m}$-thick garnet films indicates an out-of-plane magnetization component along with an existence of a cubic magnetic anisotropy axis along the [111] axes of the garnet (see Fig. 2(a)). However, for the $1.8 \mu \mathrm{m}$ garnet thickness, the hysteresis loop shows a significant remanence component (Fig. 2(b)) and a field saturation near 200 Oe that is four times lower than for the 5.8- $\mu$ m-thick garnet-only film (Figs. 2(a) and 2(b)). A clear difference in the magnetization reversal process with and without a $2 \mathrm{~nm}$ cover Co layer on 1.8 - $\mu$ m-thick garnet film was observed in the field range near the coercivity (see Fig. 2(d)). In this case, one can assume that the reduction of the garnet thickness leads to a change of the effective magnetic anisotropy field and, consequently, to a change of the magnetization reversal process. As observed before, the magnetic anisotropy of a Co-substituted garnet has two contributions: ${ }^{9}$ magnetocrystalline cubic and growth-induced uniaxial ones.

To confirm the modification of the anisotropy, measurements were performed at room temperature using both a magneto-optical-based magnetometer and a ferromagnetic resonance (FMR) X-band spectrometer. The resonance field
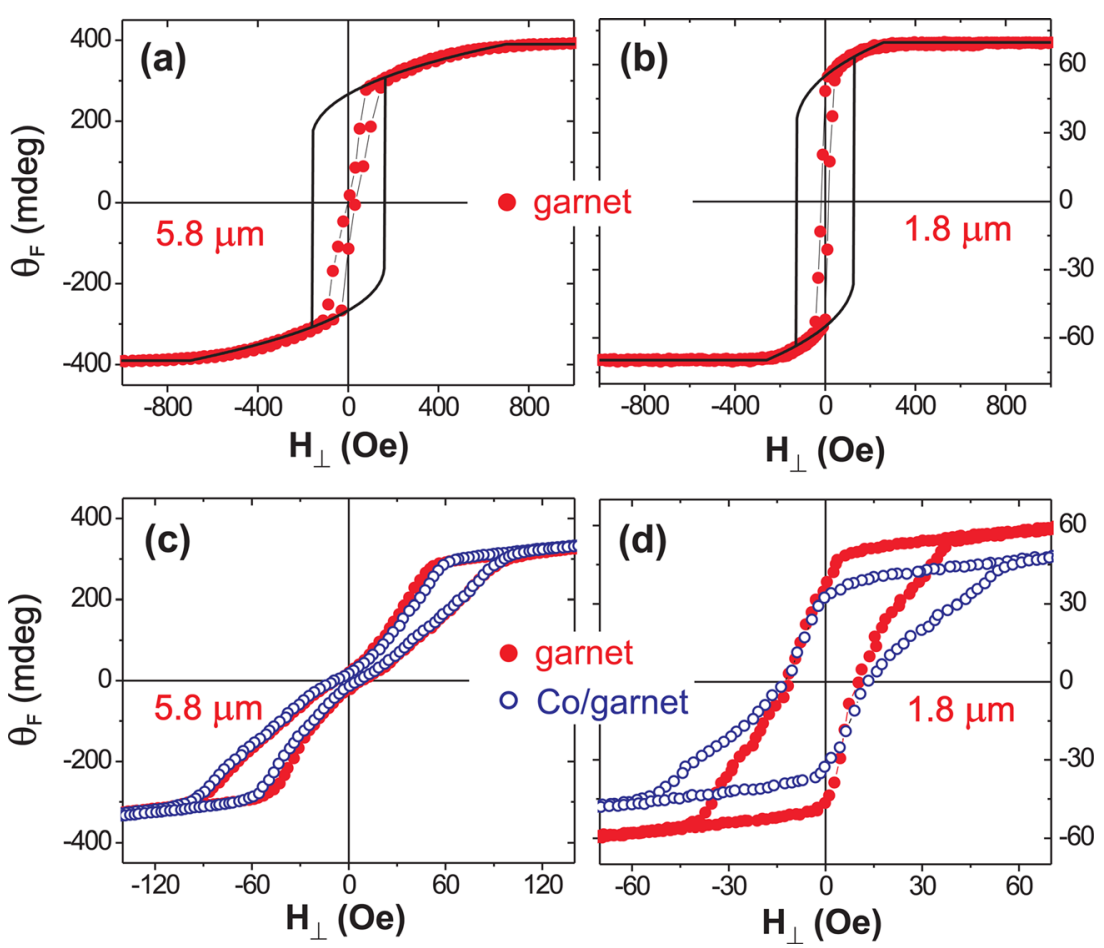

FIG. 2. (Color online) Hysteresis loops measured as Faraday rotation for $5.8 \mu \mathrm{m}$ (a) and $1.8 \mu \mathrm{m}$ (b) garnet thicknesses. The loops recorded in small magnetic field range are shown for both garnet-only (full points) and $2 \mathrm{~nm}$ $\mathrm{Co} /$ garnet (open points) films for $5.8 \mu \mathrm{m}$ (c) and $1.8 \mu \mathrm{m}$ (d) garnet thicknesses. Solid lines are fitted using Eq. (1) and following values of anisotropy constants: (a) $\mathrm{K}_{1}=-2 \times 10^{3} \mathrm{erg} / \mathrm{cm}^{3}$ and $\mathrm{K}^{\mathrm{YIG}}=-0.1 \times 10^{3} \mathrm{erg} / \mathrm{cm}^{3}$; (b) $\mathrm{K}_{1}=-2 \times 10^{3} \mathrm{erg} / \mathrm{cm}^{3}$ and $\mathrm{K}^{\mathrm{YIG}}{ }_{\mathrm{u}}=1 \times 10^{3} \mathrm{erg} / \mathrm{cm}^{3}$. 
$\mathrm{H}_{\mathrm{R}}$ (Ref. 15) was determined for different polar $\theta_{\mathrm{H}}$ and inplane azimuthal $\varphi_{\mathrm{H}}$ angles measured from the film normal and [100] direction in the sample plane, respectively. Measured values of $\mathrm{H}_{R}$ are related to the magnetic anisotropy constants and enable the determination of the easy magnetization axes as minima in $\mathrm{H}_{R}$. The experimental dependence of the $\mathrm{H}_{\mathrm{R}}$ field as a function of the angles $\theta_{\mathrm{H}}$ and $\varphi_{\mathrm{H}}$ for the $\mathrm{Co} /$ garnet sample is shown in Fig. 3 (dots). For $\mathrm{Co} /$ garnet heterostructures and both garnet thicknesses, two resonance lines were observed in the FMR spectra. In this way, the Co layer and garnet film contributions to the FMR line were separated. The existence of easy magnetization axes along the $\langle 111\rangle$ directions for the garnet contributions

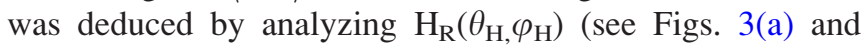
$3(\mathrm{c})$ ). This result correlates well with the Faraday experiments for garnet-only film, shown in Fig. 2(a). For the $2 \mathrm{~nm}$ Co layer, the easy magnetization axis lies in the sample plane (Fig. 3(b)) and is also connected with the in-plane anisotropy.

A qualitative analysis of the FMR and magnetization curves gives rise to the following description of the magnetic anisotropy energy $\mathrm{E}_{\mathrm{A}}$, which contains cubic, growth-induced, and in-plane uniaxial anisotropies:

$$
\begin{aligned}
E_{A}(\vec{m}, \vec{M})= & K_{1}\left[\left(m_{x} m_{y}\right)^{2}+\left(m_{x} m_{z}\right)^{2}+\left(m_{z} m_{y}\right)^{2}\right] \\
& +K_{e f f}^{Y I G}\left[1-m_{z}^{2}\right]^{2}+K_{e f f}^{C o}\left[1-M_{z}^{2}\right]^{2}
\end{aligned}
$$

where $\mathrm{m}_{\mathrm{i}}$ is the normalized components of magnetization vector in garnet defined by polar and azimuthal angles $\theta$ and $\varphi$, respectively ( $\varphi$ is measured from the [100] garnet direction), $\mathrm{M}_{\mathrm{z}}$ is the normalized perpendicular component of magnetization vector in the Co layer defined by the polar angle $\theta, \mathrm{K}_{1}$ is the cubic anisotropy constant of garnets, $\mathrm{K}^{\mathrm{YIG}}$ eff is the effective growth-induced anisotropy constant for the garnet film, and
$\mathrm{K}^{\mathrm{Co}}$ eff is the effective uniaxial anisotropy constant for a $2 \mathrm{~nm}$ Co layer. The bulk value of saturation magnetization $1420 \mathrm{G}$ was assumed for the $2 \mathrm{~nm}$ Co thickness. Magnetic anisotropy constants were fitted using Eq. (1) and standard FMR conditions ${ }^{15}$ for each resonance line of YIG and ultrathin Co films. Solid lines in Fig. 3 show the results of the fitting procedure.

Thus, let us analyze, step-by-step, the magnetic anisotropy constants of the Co layer and garnet films from FMR field and magnetization loops. First, for the Co layer deposited on the garnet films of both thicknesses, the effective anisotropy constant is $\mathrm{K}^{\mathrm{Co}}$ eff $=-9.9 \times 10^{6} \mathrm{erg} / \mathrm{cm}^{3}$, and that corresponds to the case of in-plane magnetic anisotropy in the Co layer (see Fig. 3(b)). Second, from the part of the FMR spectrum, which corresponds to garnet-only films, the cubic $\mathrm{K}_{1}=-2 \times 10^{3} \mathrm{erg} / \mathrm{cm}^{3}$ anisotropy contribution for both 1.8 and $5.8 \mu \mathrm{m}$ garnet thickness were deduced from the fitting procedure, assuming the saturation magnetization $\mathrm{M}_{\mathrm{s}}=7 \mathrm{G} .{ }^{16}$ In the case of negative $\mathrm{K}_{1}$, four easy magnetization-axis orientations along a $\langle 111\rangle$-type of crystallographic direction to the sample plane in garnet films exist. Third, cubic constants $\mathrm{K}_{1}$ determined by FMR techniques were used for the fitting of the Faraday loops measured for 1.8 and $5.8 \mathrm{~mm}$ garnet parts of a sample. In this case, the effective growth-induced uniaxial anisotropy constants $\mathrm{K}^{\mathrm{YIG}}{ }_{\text {eff }}=\mathrm{K}_{\mathrm{u}}^{\mathrm{YIG}}-2 \pi \mathrm{M}^{2}$ for different garnet thicknesses were determined from the fitted Faraday hysteresis loops (solid lines in Figs. 2(a) and 2(b)) within the coherent magnetization rotation model (i.e., assuming a single-domain state). Typically, the behavior of fitted curves is in agreement with the experiments, excluding the region near coercivity, where magnetic domains dominate the reversal behavior.

After separation of the demagnetization contribution from the uniaxial anisotropy, the anisotropy constant $\mathrm{K}_{\mathrm{u}}^{\mathrm{YIG}}$ for two thicknesses of garnet films was obtained. Before etching, in the sample with $5.8 \mu \mathrm{m}$ layer thickness,
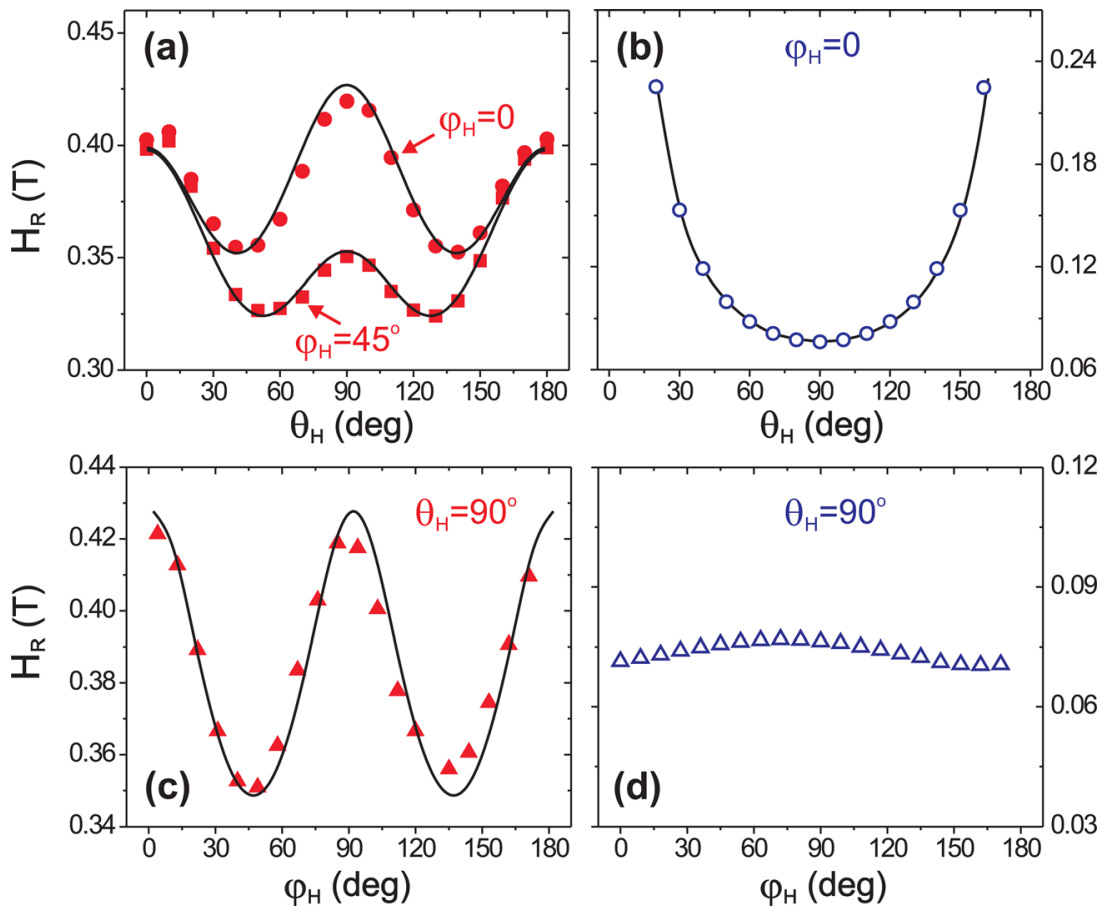

FIG. 3. (Color online) The polar (for $\varphi_{\mathrm{H}}=0$ and $45^{\circ}$ ) and azimuthal (for $\theta_{\mathrm{H}}=90^{\circ}$ ) dependence $\mathrm{H}_{\mathrm{R}}$ for $\mathrm{Co} /$ garnet sample after separation contributions from garnet parts (a), (c) and $2 \mathrm{~nm}$ Co (b), (d) layer. The dots are experimental values, and solid lines were fitted experimental results using Eq. (1). 
the growth-induced uniaxial anisotropy constant was measured to be $\mathrm{K}_{\mathrm{u}}^{\mathrm{YIG}}=-0.1 \times 10^{3} \mathrm{erg} / \mathrm{cm}^{3}$, which is smaller than cubic contribution. In this case, the hysteresis loop in Fig. 2(a) is typical for the case of cubic magnetic symmetry. The etching of the garnet film to reduce its thickness does not lead to any modification of the film composition. ${ }^{17}$ Therefore, the main factor changing the magnetization reversal behavior and the magnetic anisotropy should be the effective perpendicular growth-induced contribution. As a result, for the $1.8 \mu \mathrm{m}$ thickness, $\mathrm{K}_{\mathrm{u}}^{\mathrm{YIG}}$ has a positive sign and is equal to $1 \times 10^{3} \mathrm{erg} / \mathrm{cm}^{3}$. The existence of the out-of-plane component is consistent with the observation of non-zero remanence in the hysteresis loop, as shown in Fig. 2(b). As a result, the competition of cubic and growth-induced contributions produces strong changes of magnetic behavior of the heterostructure.

The magnetostatic coupling between the Co overlayer and the garnets of different thicknesses can be clearly seen by comparison of the small field behavior of the hysteresis loops, shown in Figs. 2(c) and 2(d). The influence of this coupling on the magnetization reversal is visible along with a strong modification of this behavior for the $\mathrm{Co} / 1.8 \mu \mathrm{m}$ garnet structure (see Fig. 2(d)). Such a magnetization reversal process is possibly related to the specific magnetic domain structure. To confirm this, the observation of the magnetic domain structures was performed using an optical polarizing microscope via the Faraday effect. A Carl Zeiss Jenapol optical microscope was equipped with a Xe lamp and a CoolSNAP Princeton Instruments high-sensitivity camera having a $1300 \times 1030$ pixels, cooled CCD element. The magnetic contrast of the domain structure was improved by standard image processing. The following procedure was used for magnetic domain generation and visualization; the sample was saturated in a sufficiently large $\mathrm{H}_{\perp}$ field, and the reference image was recorded. In the next step, images were registered in real time while continuously varying $\mathrm{H}_{\perp}$. After image processing, each image is the result of the difference between the domain structure in a given magnetic field and the reference image. Stripe domain structures are observed, both in garnet-only and $\mathrm{Co} /$ garnet structures. The images of domain structure for the $5.8-\mu \mathrm{m}$-thick garnet part was recorded at $\mathrm{H}=0$ and $\mathrm{H}=40$ Oe, as shown in Figs. 4(a) and 4(b). In this case, the preference direction of the domain
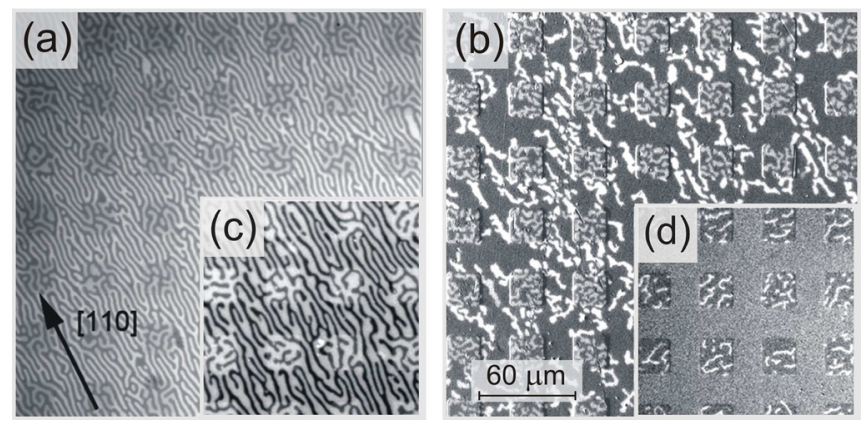

FIG. 4. (Color online) Images of magnetic domain structure in $2 \mathrm{~nm}$ $\mathrm{Co} /$ garnet pattern area recorded at zero magnetic field for: $5.8 \mu \mathrm{m}$ (a) and $1.8 \mu \mathrm{m}$ (b) garnet thickness. Insets show the domain structure images recorded in $\mathrm{H}_{\perp}=40$ Oe for: $5.8 \mu \mathrm{m}$ (c) and $1.8 \mu \mathrm{m}$ (d). walls and the parallel-stripe, indicating the orientation of the easy magnetization axis, is along [110]. However, in the magnetostatically coupled $\mathrm{Co} / 5.8 \mu \mathrm{m}$ garnet, the domain structure is changed to a labyrinth-like pattern (see Fig. 4(a)). In addition, the process of magnetization reversal in the $\mathrm{Co} / 5.8 \mu \mathrm{m}$ garnet parts is very similar to that of the garnet-only parts in both the period of the stripes (see Figs. 4(a) and 4(c)) and the values of the saturation field of about 100 Oe (see inset of Fig. 2(c)). The geometry of the domain structure on both parts of the $1.8-\mu \mathrm{m}$-thick garnet is different. For the bare $1.8 \mu \mathrm{m}$ garnet, during the application of an external magnetic field, the dendritic-like domains gradually reduce their length and disappear at $\mathrm{H}_{\perp}=40 \mathrm{Oe}$ (see Fig. 4(d)). The period and the domain size in the Co/garnet structure is less than in a pure garnet film (Fig. 4(b)). In this case, the value of the saturation field is noticeably increasing to 55 Oe (see Fig. 2(d)). In the inset of Fig. 4(b), the presence of $\mathrm{Co} /$ garnet domains on a monodomain background area is well visible.

\section{SUMMARY}

In summary, we observed that the magnetic anisotropy and magnetic domain structure of $\mathrm{Co} /$ garnet heterostructures can be tuned by varying the garnet thickness from 5.8 to $1.8 \mu \mathrm{m}$ and adding a Co cover layer. The $1.8 \mu \mathrm{m}$ garnet thickness has a strong effect on the perpendicular growthinduced magnetic anisotropy. The shape of the domains could be explained by a competition between cubic and perpendicular magnetic anisotropy in both garnet films. In addition, the modification of the domain structure due to the magnetostatic coupling has been demonstrated. In principle, depositing ultrathin ferromagnetic layers on a garnet film can also lead to new effects in magnetization dynamics, due to the influence of the effective magnetic field of the ferromagnetic layer and/or the coupling between ferromagnetic layer and garnet.

\section{ACKNOWLEDGMENTS}

The authors thank R. Gieniusz for analysis of FMR results. This work was supported by European Community's Seventh Framework Programme FP7/2007-2013 under Grant Agreement No. 214810 (FANTOMAS).

${ }^{1}$ V. I. Belotelov, I. A. Akimov, M. Pohl, V. A. Kotov, S. Kasture, A. S. Vengurlekar, A. V. Gopal, D. R. Yakovlev, A. K. Zvezdin, and M. Bayer, Nature Nanotechnol. 6, 370 (2011).

${ }^{2}$ A. V. Chumak, V. S. Tiberkevich, A. D. Karenowska, A. A. Serga, J. F. Gregg, A. N. Slavin, and B. Hillebrands, Nature Commun. 1, 141 (2010).

${ }^{3}$ S. O. Demokritov, V. E. Demidov, O. Dzyapko, G. A. Melkov, A. A. Serga, B. Hillebrands, and A. N. Slavin, Nature 443, 430 (2006).

${ }^{4}$ A. B. Chizhik, I. I. Davidenko, A. Maziewski, and A. Stupakiewicz, Phys. Rev. B 57, 14366 (1998).

${ }^{5}$ F. Hansteen, A. V. Kimel, A. Kirilyuk, and Th. Rasing, Phys. Rev. Lett. 95, 047402 (2005).

${ }^{6}$ Y. S. Chun and K. M. Krishnan, J. Appl. Phys. 95, 6858 (2004).

${ }^{7}$ N. Vukadinovic, J. Ben Youssef, V. Castel, and M. Labrune, Phys. Rev. B 79, 184405 (2009).

${ }^{8}$ A. Maziewski, J. Magn. Magn. Mater. 88, 325 (1990).

${ }^{9}$ M. Tekielak, A. Stupakiewicz, A. Maziewski, and J. M. Desvignes, J. Magn. Magn. Mater. 254-255, 562 (2003). 
${ }^{10}$ A. Stupakiewicz, A. Maziewski, I. Davidenko, and V. Zablotskii, Phys. Rev. B 64, 064405 (2001).

${ }^{11}$ F. Atoneche, A. M. Kalashnikova, A. V. Kimel, A. Stupakiewicz, A. Maziewski, A. Kirilyuk, and Th. Rasing, Phys. Rev. B 81, 214440 (2010). ${ }^{12}$ P. Görnert, M. Nevřiva, J. Šimšová, W. Andrä, W. Schüppel, P. Šumšál, and R. Bubáková, Phys. Status Solidi A 74, 107 (1982).

${ }^{13}$ G. Winkler, Magnetic Garnets (Friedr. Vieweg \& Sohn, Braunschweig, 1981).
${ }^{14}$ A. I. Stognij, V. F. Meshcheryakov, N. N. Novitskii, F. Fettar, and M. V. Pashkevich, Tech. Phys. Lett. 35, 6 (2009).

${ }^{15}$ M. Farle, Rep. Prog. Phys. 61, 755 (1998).

${ }^{16}$ R. Jabłonski, A. Maziewski, M. Tekielak, and J. M. Desvignes, J. Magn. Magn. Mater. 160, 367 (1996).

${ }^{17}$ A. I. Stognii, Ph.D. thesis, Institute of Solid State and Semiconductor Physics, National Academy of Sciences of Belarus, 1993. 